Post-print of: Plant Mol Biol (2011) 77:529-531

\title{
DNA methylation at tobacco telomeric sequences
}

María I. Vaquero-Sedas (1) and Miguel A. Vega-Palas (1)

(1) Instituto de Bioquímica Vegetal y Fotosíntesis, Universidad de Sevilla, CSIC, Seville, Spain

\begin{abstract}
Majerová et al. (Plant Mol Biol, 2011) have recently reported that a considerable fraction of cytosines at tobacco telomeres is methylated. Although the data presented in this report indicate that tobacco telomeric sequences undergo certain levels of DNA methylation, it is not clear whether the methylated sequences are at telomeres, at internal chromosomal loci or at both.
\end{abstract}

\section{Keywords}

Nicotiana tabacum, Arabidopsis thaliana, Telomeres, Subtelomeres, ITSs, Bisulfite treatment, Euchromatin, Heterochromatin

Dear Editor,

We have read with interest the article entitled "Hypomethylating drugs efficiently decrease cytosine methylation in telomeric DNA and activate telomerase without affecting telomere lengths in tobacco cells", which has been recently published in Plant Molecular Biology by Majerová et al. (2011). The authors of this article analyzed telomeric DNA methylation in tobacco BY-2 cells by bisulfite cytosine conversion. DNA treatment with bisulfite is known to convert unmethylated cytosines to uracyl. In contrast, methylated cytosines remain unchanged after incubation with bisulfite. Thus, amplification and sequencing of bisulfite treated DNA reveals methylated cytosines. Majerová and colleagues subjected tobacco BY-2 cells, treated or untreated with demethylating agents, to bisulfite cytosine conversion. Then, they purified the resulting DNA samples and, instead of analyzing them by sequencing, analyzed them by hybridization. For that purpose, they dot-blotted the DNA samples and sequentially hybridized them with a telomeric probe (AtTR4-C; 5'CCCTAAACCCTAAACCCTAAACCCTAAA-3') and with a degenerated telomeric probe (DEGENER; 5'-TTAGRRTTTAGRRTTTAGRRTTTAGRRT-3', where $R$ is $G$ or A) (Majerová et al. 2011; Vrbsky et al. 2010). As a control, Majerová and colleagues also hybridized Arabidopsis genomic DNA untreated with bisulfite. Since tobacco telomeres are composed of tandem arrays of the Arabidopsis type telomeric repeat $\left(5^{\prime}\right.$-CCCTAAA- $\left.3^{\prime}\right)$, the AtTR4-C probe served as a loading amount normalization control (Richards and Ausubel 1988; Suzuki et al. 1994). In turn, the DEGENER probe revealed the relative levels of methylation in the third cytosine of the 
telomeric sequence $5^{\prime}$-CCCTAAA-3', which is the major type of methylation found in this sequence in Arabidopsis. The first and second cytosines of the 5'-CCCTAAA-3 sequence undergo lower levels of methylation (Cokus et al. 2008). Majerová and colleagues found that the ratios of DEGENER/AtTR4-C hybridization signals obtained with bisulfite treated cells were lower than the ratio observed with the control DNA sample untreated with bisulfite. In addition, they also found that the ratios obtained when the BY-2 cells were treated with demethylating agents were lower than the ratio obtained with untreated cells. Based on these results, they concluded that a considerable fraction of cytosines is methylated in telomeric DNA of tobacco BY-2 cells. In our opinion, this conclusion should be further supported with additional experiments. It is not clear whether the methylated cytosines are telomeric. They could also be at Interstitial Telomeric Sequences (ITSs) present in subtelomeric regions or at more internal loci.

Interstitial telomeric sequences can be perfect or degenerated and have been found in a wide variety of eukaryotic organisms (Meyne et al. 1990; Richards et al. 1991; Gámez-Arjona et al. 2010). One of these organisms is Arabidopsis thaliana (Gámez-Arjona et al. 2010; Richards and Ausubel 1988; Richards et al. 1991; Regad et al. 1994; Uchida et al. 2002). In Arabidopsis, the amount of ITSs is so high that, when Arabidopsis genomic DNA is hybridized with a telomeric probe, most of the resulting hybridization signals correspond to ITSs (Gámez-Arjona et al. 2010). Thus, in principle, Arabidopsis telomeric chromatin structure analyses based on hybridization with a telomeric probe mainly reflect the chromatin structure of ITSs. Majerová and colleagues referred in their article that Arabidopsis telomeres exhibit high levels of heterochromatic marks like H3K92Me, H3K27Me and DNA methylation. In our opinion, this statement is not accurate because it is based in a previous publication in which Arabidopsis telomeres and ITSs were simultaneously analyzed by hybridization with a telomeric probe (Vrbsky et al. 2010). Therefore, these studies reflected mainly the chromatin structure of ITSs. We have previously described a simple way to independently analyze the chromatin structure of Arabidopsis telomeres and ITSs (Vaquero-Sedas et al. 2010). By following this procedure, we found that Arabidopsis telomeres exhibit euchromatic features, including low levels of H3K92Me, H3K27Me and DNA methylation. These and other telomeric chromatin structure studies have led us to emphasize the necessity to analyze the chromatin structures of telomeres and ITSs independently (Vaquero-Sedas and Vega-Palas 2011).

Perfect and degenerated ITSs that can hybridize with a telomeric probe are also present in the genome of tobacco, although their relative amount with regard to telomeres is smaller than in Arabidopsis (Fajkus et al. 1995b; Horáková and Fajkus 2000; Kenton et al. 1993; Suzuki et al. 1994). Several lines of evidence lead to this conclusion. As mentioned above, telomeres in tobacco are composed of tandem arrays of telomeric repeats that are presumably perfect and, therefore, remain uncut after restriction enzymes digestion. Tobacco telomeres localize within DNA fragments longer than $20 \mathrm{~kb}$ after digestion with different restriction enzymes (Suzuki et al. 1994). However, when tobacco genomic DNA is digested with the frequently cutting restriction enzyme Haelll and hybridized with a telomeric probe, hybridization signals smaller than $2 \mathrm{~kb}$ are displayed (Fajkus et al. 1995b). These signals correspond to low molecular weight DNA fragments containing telomeric sequences and reflect the existence of ITSs in tobacco. Tobacco ITSs have been also revealed by DNA sequencing and by cytogenetics (Horáková and Fajkus 2000; Kenton et al. 1993; Suzuki et al. 1994). Internal telomeric signals can be displayed 
by in situ hybridization studies of tobacco cells using a telomeric probe. However, most of the signals detected in these studies correspond to telomeres (Kenton et al. 1993). These results do not necessarily imply a very low abundance of ITSs in tobacco because in situ hybridization studies are not expected to display short stretches of ITSs. Micrococcal nuclease assays might have also revealed the presence of ITSs in tobacco. When tobacco nuclei preparations are digested with increasing amounts of micrococcal nuclease and the resulting DNA samples are resolved in an agarose gel, a well-defined nucleosome ladder of about $180 \mathrm{bp}$ can be observed after staining with ethidium bromide (Fajkus et al. 1995a). If this DNA ladder is transferred to a membrane and hybridized with a specific subtelomeric probe (HRS60), a similar well-defined ladder of about $180 \mathrm{bp}$ is observed. However, if the same membrane is hybridized with a telomeric probe, the resulting micrococcal nuclease ladder is shorter, about $157 \mathrm{bp}$, which reflect the typical nucleosome spacing of eukaryotic telomeres. Interestingly, the tobacco telomeric ladder generated by micrococcal nuclease is smeared and ill-defined whereas telomeric ladders from other plants are well-defined (Fajkus et al. 1995a; Vershinin and Heslop-Harrison 1998). The low definition of the tobacco telomeric ladder could be due to the existence of ITSs that fold into longer nucleosomes than the average telomeric nucleosomes, as previously described in Arabidopsis (Gámez-Arjona et al. 2010).

As proposed by Majerová and colleagues, tobacco telomeres could certainly undergo DNA methylation. However, we believe that this conclusion should be further supported by additional studies that evaluate the contribution of tobacco ITSs to the hybridization signals obtained with the DEGENER probe. If the signals obtained with this probe corresponded only to telomeres, we agree that tobacco telomeres undergo certain level of DNA methylation. Otherwise, this conclusion would not be clear and other scenarios could be possible, including the one found in Arabidopsis. In Arabidopsis, whereas telomeres exhibit euchromatic features, ITSs are heterochromatic. Thus, Arabidopsis ITSs have, as an average, higher levels of cytosine methylation than telomeres although telomeres could still have some methylated cytosines. If tobacco telomeres were not methylated at all, they would be expected to hybridize very inefficiently with the DEGENER probe (the percentage of identity between telomeres and the probe would be about $71 \%$ ). In contrast, short arrays of tobacco methylated ITSs could hybridize more efficiently with this probe and be responsible for most of the signal obtained. In this case, the ratio of DEGENER/AtTR4-C hybridization signals would also be expected to decrease after treatment with demethylating agents. 


\section{References}

Cokus S, Feng S, Zhang X, Chen Z, Merriman B, Haudenschild C, Pradhan S, Nelson S, Pellegrini $M$, Jacobsen $S$ (2008) Shotgun bisulphite sequencing of the Arabidopsis genome reveals DNA methylation patterning. Nature 452:215-219

Fajkus J, Kovarík A, Královics R, Bezdek M (1995a) Organization of telomeric and subtelomeric chromatin in the higher plant Nicotiana tabacum. Mol Gen Genet 247:633-638

Fajkus J, Královics R, Kovaríc A, Fajkusová L (1995b) The telomeric sequence is directly attached to the HRS60 subtelomeric tandem repeat in tobacco chromosomes. FEBS Lett 364:33-35

Gámez-Arjona F, López-López C, Vaquero-Sedas M, Vega-Palas M (2010) On the organization of the nucleosomes associated with telomeric sequences. Biochim Biophys Acta 1803:1058-1061

Horáková M, Fajkus J (2000) TAS49-a dispersed repetitive sequence isolated from subtelomeric regions of Nicotiana tomentosiformis chromosomes. Genome 43:273-284

Kenton A, Parokonny A, Gleba Y, Bennett M (1993) Characterization of the Nicotiana tabacum L. genome by molecular cytogenetics. Mol Gen Genet 240:159-169

Majerová E, Fojtová M, Mozgová I, Bittová M, Fajkus J (2011) Hypomethylating drugs efficiently decrease cytosine methylation in telomeric DNA and activate telomerase without affecting telomere lengths in tobacco cells. Plant Mol Biol. doi:10.1007/s11103-011-9816-7

Meyne J et al (1990) Distribution of non-telomeric sites of the (TTAGGG)n telomeric sequence in vertebrate chromosomes. Chromosoma 99:3-10

Regad F, Lebas M, Lescure B (1994) ITSs within the Arabidopsis thaliana genome. J Mol Biol 239:163-169

Richards E, Ausubel F (1988) Isolation of a higher eukaryotic telomere from Arabidopsis thaliana. Cell 53:127-136

Richards E, Goodman H, Ausubel F (1991) The centromere region of Arabidopsis thaliana chromosome 1 contains telomere-similar sequences. Nucleic Acids Res 19:3351-3357

Suzuki K, Yamagiwa Y, Matsui T, Yoshida K (1994) Restriction enzyme-resistant high molecular weight telomeric DNA fragments in tobacco. DNA Res 1:129-138

Uchida W, Matsunaga S, Sugiyama R, Kawano S (2002) Interstitial telomere-like repeats in the Arabidopsis thaliana genome. Genes Genet Syst 77:63-67

Vaquero-Sedas M, Vega-Palas M (2011) On the chromatin structure of eukaryotic telomeres. Epigenetics 6:1055-1058

Vaquero-Sedas M, Gámez-Arjona F, Vega-Palas M (2010) Arabidopsis telomeres exhibit euchromatic features. Nucleic Acids Res. doi:10.1093/nar/gkq1119

Vershinin A, Heslop-Harrison J (1998) Comparative analysis of the nucleosomal structure of rye, wheat and their relatives. Plant Mol Biol 36:149-161 
Vrbsky J, Akimcheva S, Watson J, Turner T, Daxinger L, Vyskot B, Aufsatz W, Riha K (2010) siRNA-mediated methylation of Arabidopsis telomeres. PLoS Genet 6:e1000986 